D.T. Wickramasinghe, L. Ferrario, and G.V. Bicknell, eds.

\title{
Dissipation of Turbulent Magnetic Fields In Accreting Black Holes
}

\author{
Victor Kowalenko \\ School of Physics, University of Melbourne, Parkville Victoria 3052, \\ Australia
}

Fulvio Melia

University of Arizona, Tucson, AZ 85721, USA

\begin{abstract}
Calculations of the spectrum resulting from accretion onto a massive black hole often make use of the "equipartition assumption" in order to estimate the magnetic field intensity. Thus, the mechanism for the dissipation of the magnetic field and the resulting dynamical influence on the gas have not been treated quantitatively, nor self-consistently. Here, we introduce an alternative approach for modelling the magnetic field dissipation from basic principles, using current ideas about turbulent fields and tearing mode instabilities.
\end{abstract}

\section{Introduction}

In a recently developed model for the spectrum of the galactic center black hole candidate $\mathrm{Sgr} \mathrm{A}^{*}$ (Melia 1994), the overall emissivity is determined by a combination of electron-electron and electron-ion bremsstrahlung, as well as cyclotron/synchrotron radiation by thermal electrons. The latter dominates the flux at radio frequencies, whereas the former contributes to the spectrum primarily at UV and higher energies. However, to calculate the "magnetic bremsstrahlung" emissivity, one often invokes the equipartition assumption because the energy density of a frozen-in magnetic field in a radially-converging flow increases inward much more rapidly than the gravitational energy. Thus, any magnetic field carried by the highly-ionized accreting matter should find a way to decrease its energy density to remain at the same order as the other energy components, for it would otherwise not remain anchored or frozen into the gas. Hence, the usual assumptions made in studying accretion onto a massive black hole are that the magnetic energy density is a constant factor times the gravitational potential energy density and that the difference between these two energy densities is dissipated and added as heat to the accreting matter.

Here we aim to set up the framework required for a future study of magnetic field dissipation and turbulence carried by the accreting plasma in order to examine what effect dispensing with the equipartition assumption has on the spectrum of an accreting black hole. 


\section{Calculational Method}

The description provided here is a necessarily abbreviated account reported with significantly more detail in Kowalenko \& Melia (1996). We begin by examining the essential characteristics of the simplest form of turbulence known as isotropic turbulence (Hinze 1959), whose character is determined by the ratio of the inertial forces over the viscous forces (i.e., the Reynolds number). For the accreting black hole model described above, we are primarily concerned with high Reynolds numbers, where in a fully developed state of turbulence it is not the largest eddies that possess the maximum kinetic energy but rather the eddies in a higher range of wavenumbers known as the range of energy-containing eddies. This range provides the main contribution to the energy spectrum and within it the spectrum follows the famous Kolmogorov power-law. In recent work, Giacolone \& Jokipii (1994) have introduced a novel numerical method for simulating such a turbulent magnetic field by writing the total field intensity as the sum over a given number of transverse waves of random polarisation, with the condition that it satisfies the divergence theorem, and that it is scaled by the total energy density. The calculation is carried out on a lattice of wavenumbers chosen with a spacing that covers the inertial subrange of the range of energy-containing eddies discussed above.

Reconnection occurs within the plasma in locations where the field is sheared. One well-studied mechanism by which this takes place is resistive magnetic tearing, sometimes referred to as the tearing instability, which results in rapid field reconnection driven by the free energy of the sheared magnetic field (Van Hoven 1979). It occurs in plasmas whose behaviour deviates from ideal MHD by incorporating effects due to Coulomb collisions, which result in resistive behaviour and the relative diffusion of the field and the fluid. The magnetic energy, which drives the instability, disappears in the reconnection layer as it is transformed into fluid energy. The growth rate of this instability depends on the fluid temperature and density, and on the local field strength and characteristic separation of the sheared field elements.

At each time step, dictated by how fast the accreting gas compresses, the field randomization procedure is followed by a determination of the points where the field becomes sheared, and by how much. Van Hoven's treatment of the energetics of resistive magnetic tearing can then be applied to the field to infer the local dissipation rate. In this fashion, the dissipation rate is at all times totally dependent on the physical state of the compressed gas, providing a more realistic estimate of the field than that given by the equipartition assumption, and hence yielding a more meaningful radiative spectrum to compare with the data.

\section{References}

Giacolone, J. \& Jokipii, J.R. 1994, ApJ, 430, L137

Hinze, J.O. 1959, Turbulence (McGraw-Hill: New York)

Kowalenko, V. \& Melia, F. 1996, in preparation.

Melia, F. 1994, ApJ, 426, 577

Van Hoven, G. 1979, ApJ, 232, 572. 